\title{
Road Map of Micro-Engineering and Nano-Engineering from Manufacturing and Mechanical Engineering Viewpoints*
}

\author{
Akira YABE $^{* *}$, Shigeki HIRASAWA ${ }^{* * *}$, Nobuhide KASAGI ${ }^{* * * *}$, \\ Takayuki KITAMURA*****, Eiji NAKAMACHI ${ }^{\dagger}$, Yasunari TAKANO ${ }^{\dagger \dagger}$, \\ Haruo OGAWA $^{\dagger \dagger \dagger}$, Seiichi YOKOBORI $\mathrm{I}^{\dagger \dagger \dagger}$ and Masato IKEGAWA ${ }^{* * *}$
}

\begin{abstract}
A new road map of future trends in micro-engineering and nano-engineering was established from the viewpoints of manufacturing and mechanical engineering. This road map was made by evaluating the results of questionnaires given to the leading engineers including elected trustees of the JSME, and by considering the discussions during the "International Symposium of Micro-Mechanical Engineering (ISMME2003)" held on December 2003 in Tsuchiura and Tsukuba, Japan. The micro- and nano-engineering were categorized from the manufacturing viewpoint. The road map shows the important trends in micro- and nanoengineering, which include new technological areas, as well as technical development, new manufacturing processes, and new kinds of mechanical engineering. The road map also estimates, from the engineering standpoint, the expected time for practical application and technological breakthroughs.
\end{abstract}

Key Words: Road Map, Micro-Engineering, Nano-Engineering, Information Technology, Bioengineering, Environmental Engineering

\section{Introduction}

Micro-engineering and nano-technology have recently become the leading research subjects, and many

* Received 17th February, 2004 (No. 04-4058)

** AIST Chugoku, National Institute of Advanced Industrial Science and Technology (AIST), 2-2-2 Hirosuehiro, Kure, Hiroshima 737-0197, Japan.

E-mail: yabe-akira@aist.go.jp

*** Mechanical Engineering Research Laboratory, Hitachi, Ltd., 502 Kandatsu, Tsuchiura, Ibaraki 300-0013, Japan. E-mail: hira@merl.hitachi.co.jp

**** Department of Mechanical Engineering, University of Tokyo, 7-3-1 Hongo, Bunkyo-ku, Tokyo 113-8656, Japan

***** Department of Engineering Physics and Mechanics, Kyoto University, Yoshida Honmachi, Sakyo-ku, Kyoto 6068501, Japan

$\dagger$ Department of Mechanical Engineering, Osaka Institute of Technology, 5-16-1 Omiya, Asahi-ku, Osaka 535-8585, Japan

† University of Shiga Prefecture, 2500 Hassakacho, Hikone, Shiga 522-8533, Japan

$\dagger \dagger$ Olympus Co., 2-3 Kuboyama-cho, Hachiouji, Tokyo 192 8512, Japan

††† Toshiba Co., 4-1 Ukishima-cho, Kawasaki-ku, Kawasaki, Kanagawa 210-0862, Japan research projects related to them have been established with the cooperation of industries, universities, and governments. They have been promoted worldwide and have been explored in the collaboration of many researchers of different specialized fields. The roles of mechanical engineering would be tremendously important from the viewpoint of industrial applications of nano-technology, and these fields will therefore be asked to contribute to the collaborative research.

From the manufacturing viewpoint, the trends in technical development related to micro-engineering and nanoengineering can be classified into four categories: (1) Reducing the size of systems while maintaining their functions, (2) Integrating functions to systems without changing the sizes of those systems, (3) Improving system performance by adding micro- and nano-scale function to macroscale engineering applications, and (4) Increasing the efficiency of existing macroscale products by improving the microscale factors that limit their performance. These trends are the result of the close collaboration of workers in many specialized fields related to mechanical engineering, and the contributions of those workers can be considered "micro-engineering and nano-engineering."

To stimulate collaboration among the various engi- 
neering fields and to explore the future trends of microengineering and nano-engineering, we started the Interdisciplinary Research Council (called P-SCC4 in 2002 and called P-SCC5 in 2003) of the Japanese Society of Mechanical Engineers (JSME). The Interdisciplinary Research Council comprises members of various JSME divisions: Thermal Engineering Division, Fluid Engineering Division, Bioengineering Division, and Materials and Mechanics Division. Since micro-engineering and nanoengineering includes not only application technologies but also basic researches ${ }^{(1)}$, members of the Interdisciplinary Research Council were selected from the industrial, academic, and governmental sectors. Assisting this Interdisciplinary Research Council, Yabe ${ }^{(2)}$ of the National Institute of Advanced Industrial Science and Technology (AIST) reported future trends of manufacturing and explained that one of the most critical technologies would be micro-scale machining and assembly. In this paper, which is a result of the activity of the Interdisciplinary Research Council, the road map of micro-engineering and nanoengineering is introduced from the manufacturing and mechanical engineering viewpoints. The road map shows the important trends of micro-engineering, which include new technological areas, trends in technical developments, new manufacturing processes, and new kinds of mechanical engineering. The road map also estimates, from the engineering standpoint, the time required for putting technological breakthroughs to practical application.

\section{Questionnaire for Future Trends of Micro- Engineering}

The road map of future trends in micro- and nanoengineering was made by first having members of the Interdisciplinary Research Council systematically make a table of keywords of micro- and nano-engineering. Questionnaires with that table of keywords were then sent to 450 leaders of manufacturing engineering, which included the elected trustees of the JSME. These elected trustees are the leaders of the mechanical engineering field in Japan, and 80 of them returned completed questionnaires. The road map was assembled by handling their responses statistically.

After making the table of micro- and nanoengineering keywords, we determined three viewpoints from which the relation between micro- and nanoengineering and mechanical technology might be examined and determined eleven classifications based on those viewpoints.

(A) New technological areas related to micro- and nano-engineering as industrial technology:

A-1 Information technology (IT) field,

A-2 Biomedical engineering field,

A-3 Environment and energy field.

(B) Technical development related to micro- and nano-engineering:

B-1 Reducing the size of systems while maintaining their functions,

B-2 Integrating functions to systems without changing the sizes of those systems,

B-3 Improving system performance by adding microand nano-scale functions to macroscale engineering applications,

B-4 Increasing the efficiency of existing macroscale products by improving the microscale factors that limit their performance.

(C) New manufacturing and new mechanical engineering needed for micro- and nano-engineering:

C-1 Design techniques for micro- and nano-engineering, C-2 Practical application technology using micro-scale effects for new functions,

C-3 Micro-processing and assembly technology,

C-4 Reliability, maintenance, and measurement technology for macro- and nano-engineering.

The introductory part of the questionnaire was as follows. "We would be grateful if you could complete this survey, Questionnaire on Future Trends in Microengineering and Nano-engineering. This questionnaire is intended to comprehensively examine the needs, seeds, and future prospects of micro- and nano-engineering from the aspect of mechanical engineering and manufacturing technology. Your responses will be compiled in the form of a road map that predicts the future technical developments. Our questionnaire survey is targeting industrial, academic, and government leaders who have been at the forefront of technical development in Japan. The results of the questionnaire will be circulated both at home and abroad as a micro-engineering road map from Japan.

1. We ask you first to examine the relation between micro-engineering and mechanical technology.

2. We ask you next to tell us how much time you expect, from a user or engineer standpoint, to be needed for practical application of technological breakthroughs in micro-engineering and mechanical engineering."

The road map was presented and discussed in the "International Symposium of Micro-Mechanical Engineering (ISMME2003)" held on December 2003 in Tsuchiura and Tsukuba, Japan. The selected papers of ISMME2003 will be published in the special issue on "Micro Mechanical Engineering” of JSME International Journal.

\section{Road Map of the Relationship between Micro- Engineering and Manufacturing/Mechanical En- gineering}

The questionnaire respondents' opinions regarding the future trends of the relationship between microengineering and manufacturing/mechanical engineering are summarized below from three viewpoints and in terms of three classifications of the level of importance. View- 


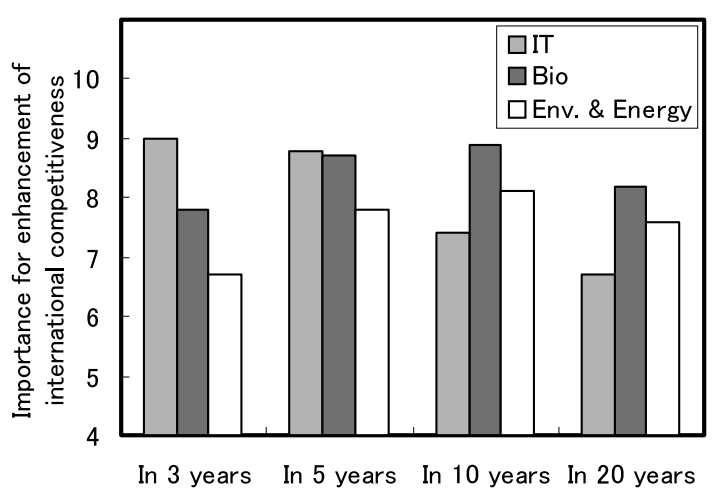

Fig. 1 Trends in the enhancement of international competitiveness of new technological areas related to micro-and nano-engineering

points:

(A) New technological areas related to micro- or nano- engineering as industrial technology,

(B) Technical development related to micro- and nano-engineering,

(C) New manufacturing and new mechanical engineering needed for micro-engineering and nanoengineering.

Classifications of the level of importance:

(a) Enhancement of international competitiveness in this field of technology,

(b) Technological breakthroughs,

(c) Contribution of technical development to industrial areas.

The results are shown in Figs. 1-6, and we could find some future trends in the results. Number of the importance at the vertical axis of Figs. 1-6 means a qualitative degree of the importance with range from 1 to 10 . We should note that the number of the returned completed questionnaires was not enough to rely on the accuracy and we analyzed the results carefully.

Figure 1 shows the trends in the enhancement of international competitiveness of new technological areas related to micro-and nano-engineering: (A)-(a). In the near future, information technology (IT) field will be more important than biomedical engineering or environment and energy fields. In 10 years, biomedical engineering field will have become increasingly important and need more research and development. The environment and energy field will show the same tendency.

Figure 2 shows the trends in the enhancement of international competitiveness due to technical development related to micro- and nano-engineering: (B)-(a). Reducing size of systems and integrating functions will be more important than adding micro- and nano-scale functions to macroscale engineering applications and increasing efficiency of existing macroscale products.

Figure 3 shows the trends in the importance of tech-

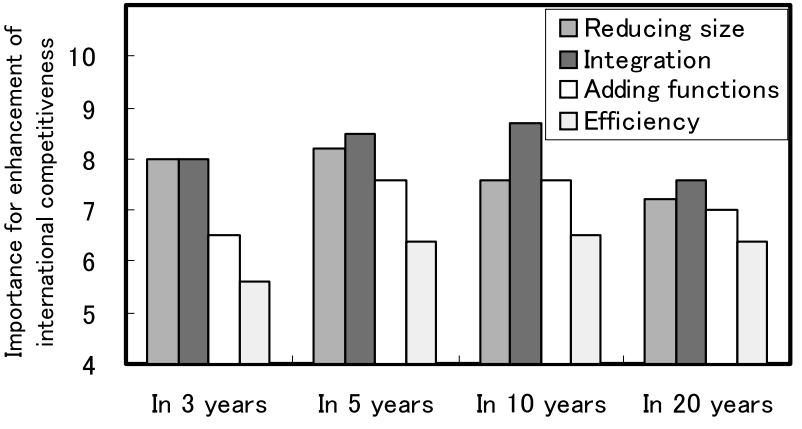

Fig. 2 Trends in the enhancement of international competitiveness due to technical development related to micro- and nano-engineering

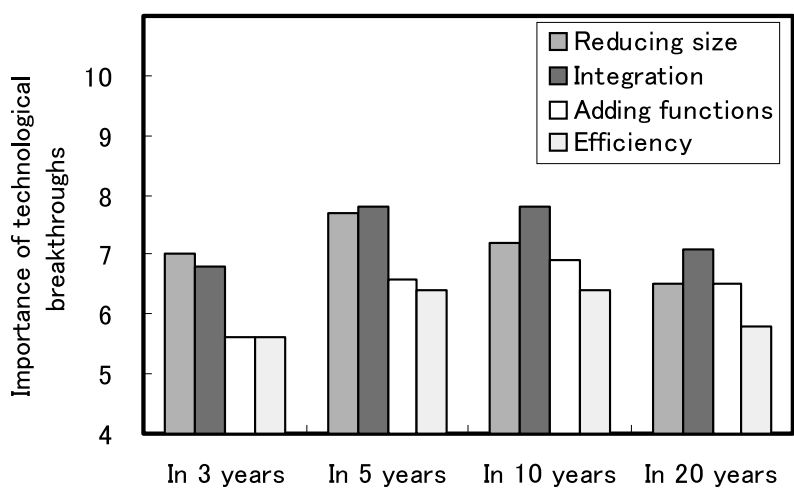

Fig. 3 Trends in the importance of technological breakthroughs in technical developments related to micro- and nanoengineering

nological breakthroughs in technical developments related to micro- and nano-engineering: (B)-(b). The importance of technological breakthroughs will increase a little slower than that of the international competitiveness of technology.

Figure 4 shows the trends in the enhancement of international competitiveness due to micro-scale mechanical engineering: (C)-(a). Nearly all aspects of microand nano-engineering were found to be equally important to the promotion of manufacturing. It is different from the earlier questionnaire ${ }^{(2)}$, in which microscale processing and assembly were found to be most important to the promotion of manufacturing .

Figure 5 shows the trends in the importance of technical breakthroughs in micro-scale mechanical engineering: (C)-(b). Nearly all aspects would have the same importance for technological breakthroughs.

Figure 6 shows the contributions of technical development to industrial areas: (B)-(c). Reducing the size of systems and integrating functions will make large contributions to the development of IT and biomedical engineering. Adding micro- and nano-scale functions to macroscale engineering applications and increasing efficiency of existing macroscale products will strengthen environment and energy field. 


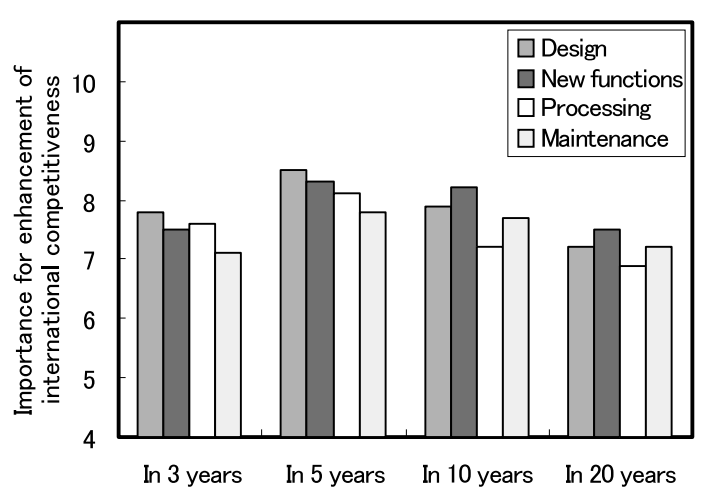

Fig. 4 Trends in the enhancement of international competitiveness due to micro-scale mechanical engineering

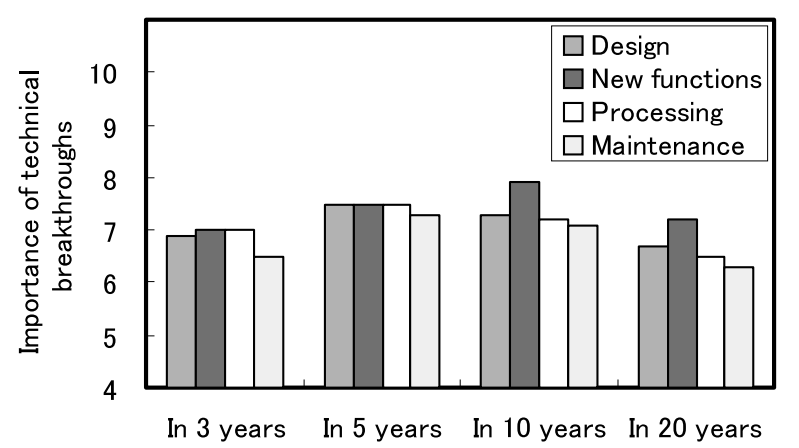

Fig. 5 Trends in the importance of technical breakthroughs in micro-scale mechanical engineering

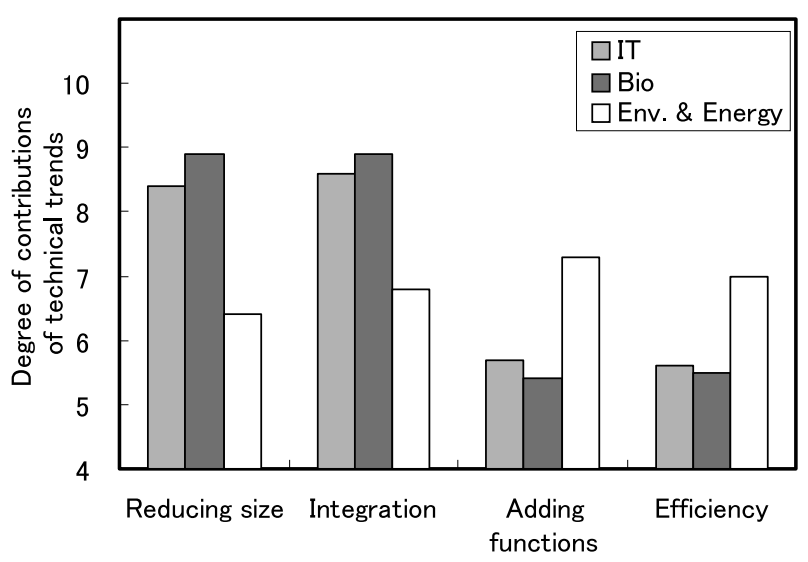

Fig. 6 Contributions of technical development to industrial areas

\section{Road Map of Expected Time for Practical Appli- cation and Technological Breakthroughs}

The questionnaire respondents' opinions regarding expectations, from the manufacturing standpoint, of the expected time for practical application and technological breakthroughs are summarized here. In this context, the time of practical application means the time needed for products to be marketed for the first time. From the 11 categories of manufacturing viewpoints, the typical keywords were selected and given in the questionnaire. Ex-

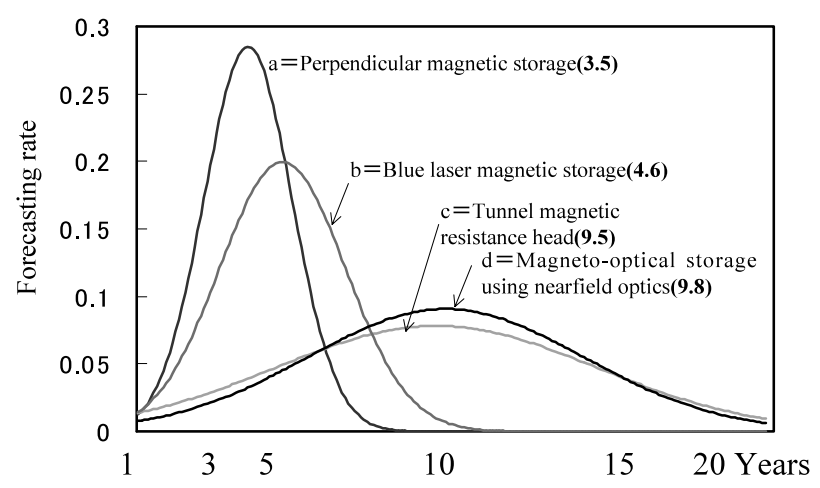

Fig. 7 Information technology (IT) field: Magnetic storage device

amples of the expected times are shown in Fig. 7 as averages with standard deviations, and the rest of the average expected times are listed in Table 1. Some trends and characteristics are evident in these results. Many sessions of ISMME2003 were related to the categories of manufacturing viewpoints, and some related keywords are also shown in the following explanations.

In Table 1(A-1) magnetic storage devices were selected as the representative of the information technology (IT) field. The standard deviations are from 1 to 5 years (Fig. 7). Major researchers in this field expected practical application related to magnetic storage devices to occur after periods shorter by about 1 year than the periods expected by researchers who were not major researchers in this field. The IT-related keywords shown in the ISMME2003 proceedings ${ }^{(3)}$ are switch-mode power supply, high-power amplifier, component packaging, activehead slider, thin-film semiconductor device, micro-lens scanner, copper interconnects, and fiber-optic telecommunication MEMS.

(A-2) Representative of the biomedical engineering field selected drug delivery. Nearly all researchers who responded to this questionnaire had not done major work in this field. Their expected times were not less than 6 years. The keywords related to this area that are shown in the ISMME2003 proceedings $^{(3)}$ are micro-moving mechanism for medical application, DNA detection device, artificial gel, and micro-fluidics.

(A-3) In the environment and energy field, catalysts and element technology using nano-technology were selected. Nearly all respondents expected practical applications to take between 5 and 10 years. This means that mechanical engineers think they should develop them within 10 years but not within 5 years. The related keywords are micro-diffusion-flame and micro-catalytic-combustor.

(B-1) Micro-sensor was selected as the representative system whose size was reducing while maintaining their functions. Major researchers in this field expected this to take 2 or 3 years longer than researchers who were not major researchers in this field expected. The related key- 
Table 1 Time expected for practical application of technological breakthroughs (Number in parentheses shows the average expected times)

(A) New technological areas

A-1 Information technology (IT) field: Magnetic storage device

\begin{tabular}{|c|c|c|c|c|}
\hline Time for practical application & In 3 years & In 5 years & In 10 years & In 20 years \\
\hline Magnetic storage device & & $a(3.5) \quad b(4.6)$ & $\begin{array}{c}\mathbf{c}(9.5) \\
\mathrm{d}(9.8)\end{array}$ & \\
\hline
\end{tabular}

A-2 Biomedical engineering field: Drug delivery

\begin{tabular}{|c|c|c|c|c|}
\hline Time for practical application & In 3 years & In 5 years & In 10 years & In 20 years \\
\hline Drug delivery & & & $\begin{array}{r}a(6.7) b(7.3) \\
c(8.5)\end{array}$ & d(12) \\
\hline
\end{tabular}

$\mathrm{c}=$ Drug delivery of drug release control, $\quad \mathrm{d}=$ Drug delivery system for cancer treatment

A-3 Environment and energy field: Catalyst and element technology using nano-technology

\begin{tabular}{|c|c|c|c|c|}
\hline Time for practical application & In 3 years & In 5 years & In 10 years & In 20 years \\
\hline $\begin{array}{l}\text { Catalyst and element technology } \\
\text { using nano-technology }\end{array}$ & & $\mathrm{a}(4.6)$ & $\begin{array}{r}\text { b(5.4) d }(6.2) \\
\mathbf{c}(5.8) \mathrm{e}(8.5) \\
\mathbf{f}(9.7)\end{array}$ & \\
\hline
\end{tabular}

(B) Trends in technical development

B-1 Reducing the size of systems while maintaining their functions

\begin{tabular}{|l|l|l|l|c|}
\hline Time for practical application & In 3 years & In 5 years & In 10 years & In 20 years \\
\hline Micro-sensor & & & $\begin{array}{c}\mathbf{a ( 5 . 1 )} \\
\text { b(6.7) }\end{array}$ & c(12) \\
\hline
\end{tabular}

$\mathrm{c}=$ Sensor for five human senses

B-2 Integrating functions to systems without changing the sizes of those systems

\begin{tabular}{|c|c|c|c|c|}
\hline Time for practical application & In 3 years & In 5 years & In 10 years & In 20 years \\
\hline Microchip & & & $\begin{array}{l}\mathrm{a}(5.6) \mathrm{c}(6.3) \\
\mathrm{b}(6.1) \mathrm{d}(8.0)\end{array}$ & e(14) \\
\hline
\end{tabular}

B-3 Improving system performance by adding micro- and nano-scale functions to macroscale engineering applications

\begin{tabular}{|c|c|c|c|c|}
\hline Time for practical application & In 3 years & In 5 years & In 10 years & In 20 years \\
\hline Heat exchanger & & & $\begin{array}{l}\mathrm{a}(5.6) \mathrm{d}(6.9) \\
\mathrm{b}(6.2) \mathrm{e}(7.4) \\
\mathrm{c}(6.3) \quad \mathrm{f}(8.8)\end{array}$ & \\
\hline
\end{tabular}

$\mathrm{a}=$ High-efficiency packaging technology for electronics,

$\mathrm{b}=$ Technology for heat transfer enhancement utilizing micro- and nano-surface configuration,

$\mathrm{c}=$ Technology for drag reduction of fluid flow utilizing micro- and nano-function,

$\mathrm{d}=$ Local high heat flux cooling technology for electronics

$\mathrm{e}=$ Thermal insulation technology utilizing nanoscale effects,

$\mathrm{f}=$ Upgrade technology for heat exchanger by utilizing nano-scale effects

words are micro-TFTCs ands MEM sensors.

(B-2) The microchip was selected as the representative of system whose size was expected to remain the same but that would have more functions. The expected times for the actual applications are beyond 5 years. The related keywords are micro-channel cooling, microfluidics, micro-fluidics device for mixing and chemical re- action, integrated micro-chemical devices, liquid-cooling, interface stabilization, micro-thermoelectric-cooler, ultramicro-scale gas-turbine system, and miniature gas turbine engine.

(B-3) Heat exchangers were selected as representative systems expected to be improved by adding microand nano-scale functions. The expected times for practi- 
tative micro-scale processing-and-assembly technology. Microscale drilling and minute-diameter-hole-penetrating are expected within 5 years. The related keywords are silicon micromachining, micro-laser-drilling, laser ablation, nanopillars, micro-droplet behavior, and micro-bonding.

(C-4) Nano-measurement was selected as the representative reliability, maintenance, and measurement technology. Improvement of figure resolution and the measurement of nano-scale properties and optical characteristics are expected in about 5 years. The related keywords are electromigration damage, mechanical properties of electroplating film, Soret effect for micro-scale mass diffusion properties, and nanoscale thermal properties using near-field optics.

\section{Summary and Conclusions}

By analyzing the questionnaire responses of elected trustees of the JSME, we made a road map of future trends in micro-engineering and nano-engineering. In the road map we selected the following three viewpoints (A), (B), and $(\mathrm{C})$ that clarified the relation between micro-/nanoengineering and mechanical/manufacturing technology.

(A) New technological areas related to micro- and nano- engineering as industrial technology:

A-1 Information technology (IT) field,

A-2 Biomedical engineering field,

A-3 Environment and energy field.

(B) Technical development related to micro- and nano-engineering:

B-1 Reducing the size of systems while maintaining their functions,

B-2 Integrating functions to systems without changing the sizes of those systems,

B-3 Improving system performance by adding microand nano-scale functions to macroscale engineering applications,

B-4 Increasing the efficiency of existing macroscale products by improving the microscale factors that limit their performance.

(C) New manufacturing and new mechanical engineering needed for micro- and nano-engineering:

C-1 Design techniques for micro- and nano-engineering,

C-2 Practical application of micro-scale effects for new functions,

C-3 Micro-processing and assembly technology,

C-4 Reliability, maintenance, and measurement technology of micro- and nano-engineering.
The road maps show the following:

(1) In the near future, information technology (IT) field will be more important than biomedical engineering or environment and energy fields. Biomedical engineering field will become more important in 10 years, as will environment and energy field.

(2) Reducing size of systems and integrating functions will be more important than adding micro- and nanoscale functions to macroscale engineering applications and increasing efficiency of existing macroscale products. Reducing size of systems and integrating functions will make large contributions to the development of IT and biomedical engineering, while environment and energy field is expected to benefit more from improving system performance by adding micro-/nano-scale functions and from increasing the efficiency of existing products by improving the microscale factors.

(3) Nearly all aspects of manufacturing-such as design, establishing new functions, processing, assembly, and measurement-are expected to be of equal importance for technological breakthroughs.

(4) Practical applications and technological breakthroughs are expected to take between 5 and 10 years for many viewpoints of micro-engineering and nanoengineering. In some aspects, the expected times differed between those who were and were not major researchers in the examined field.

We hope the road map of micro-engineering and nano-engineering will help forecast developments of macro-/nano-technologies and promote industrial applications of micro-engineering and nano-engineering. We also expect the road map will give birth new research fields in mechanical engineering.

\section{Acknowledgements}

We appreciate the elected trustees of JSME who responded to our questionnaire. We thank Prof. Hideo Yoshida of Kyoto University for helpful suggestions.

\section{References}

( 1 ) Fujie, K., The Philosophy for Technology in Research and Development, J. of JSME, (in Japanese), Vol.89, No.806 (1986), pp.79-83.

(2) Yabe, A., Nano-Technology and Thermal Engineering, Proc. Int. Symp. on Micro-Mechanical Engineering (ISMME2003), JSME, (2003), pp.31-36.

( 3 ) Proc. Int. Symp. on Micro-Mechanical Engineering (ISMME2003), JSME, (2003). 